ARTICLE

\title{
Structural snapshots of human DNA polymerase $\mu$ engaged on a DNA double-strand break
}

Andrea M. Kaminski (1D ${ }^{1}$, John M. Pryor ${ }^{2}$, Dale A. Ramsden (1) ${ }^{2}$, Thomas A. Kunkel (1) ${ }^{1}$, Lars C. Pedersen (D) ${ }^{1 凶}$ \& Katarzyna Bebenek ${ }^{1}$

Genomic integrity is threatened by cytotoxic DNA double-strand breaks (DSBs), which must be resolved efficiently to prevent sequence loss, chromosomal rearrangements/translocations, or cell death. Polymerase $\mu$ (Pol $\mu)$ participates in DSB repair via the nonhomologous end-joining (NHEJ) pathway, by filling small sequence gaps in broken ends to create substrates ultimately ligatable by DNA Ligase IV. Here we present structures of human Pol $\mu$ engaging a DSB substrate. Synapsis is mediated solely by Pol $\mu$, facilitated by single-nucleotide homology at the break site, wherein both ends of the discontinuous template strand are stabilized by a hydrogen bonding network. The active site in the quaternary Pol $\mu$ complex is poised for catalysis and nucleotide incoporation proceeds in crystallo. These structures demonstrate that Pol $\mu$ may address complementary DSB substrates during NHEJ in a manner indistinguishable from single-strand breaks.

\footnotetext{
${ }^{1}$ Genome Integrity and Structural Biology Laboratory, National Institute of Environmental Health Sciences, National Institutes of Health, 111 TW Alexander Dr. Bldg. 101/Rm F338, Research Triangle Park, NC 27709, USA. ${ }^{2}$ Curriculum in Genetics and Molecular Biology, University of North Carolina at Chapel Hill, $32-$

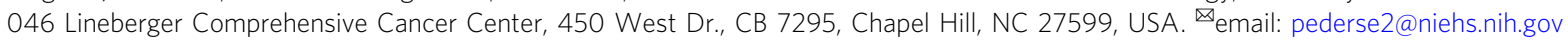


$\mathrm{G}$ enomic DNA is vulnerable to damage/breakage caused by exogenous exposures to ionizing radiation or endogenous reactive oxygen species generated through cellular metabolism $^{1}$. When phosphodiester backbone breaks on opposing DNA strands cluster, cytotoxic DNA double-strand breaks (DSBs) form. Persistently unrepaired DSBs in DNA can have disastrous consequences, leading to human cancers and other diseases ${ }^{2}$. Alternatively, DSBs can be systematically generated in a programmed manner, as observed in the $\mathrm{V}(\mathrm{D}) \mathrm{J}$ recombination pathway required for immunoglobulin gene maturation ${ }^{3}$. DSBs are repaired by multiple pathways, including nonhomologous end-joining (NHEJ), which is favored in nonreplicating cells, or in cells lacking replicated sister chromatids ${ }^{4}$. The multiprotein NHEJ complex binds and bridges broken ends so they can be ultimately rejoined $^{5}$. Its assembly is presumed to occur in a stepwise fashion, wherein the Ku70/80 heterodimer first identifies and binds the ends, in concert with the DNA-PK catalytic subunit. This core complex, known as the DNA-PK holoenzyme, enlists other protein binding factors-DNA Ligase IV, XRCC4, Artemis, XLF, and various polymerases-to subsequently process and rejoin the ends, depending on their sequence and structural composition ${ }^{5}$.

The Family X polymerases (Pols), Pol $\lambda$, Pol $\mu$, and terminal deoxyribonucleotidyl transferase (TdT) are recruited to the NHEJ complex by means of an N-terminal BRCT protein-protein interaction domain ${ }^{6}$. All three Family X enzymes are involved in $\mathrm{V}(\mathrm{D}) \mathrm{J}$ recombination, though with distinct expression and polymerization profiles. Pols $\lambda$ and $\mu$ are widely expressed, primarily template-dependent polymerases, which function in maturation of immunoglobulin heavy- and light-chain loci, respectively $^{7-9}$. In contrast, TdT expression is strictly limited to immunological cells, where it functions as a primarily templateindependent polymerase contributing to gene sequence diversity during $V(D) J$ recombination ${ }^{10}$. In addition to their specific roles in $V(D) J$ recombination, Pols $\lambda$ and $\mu$ also function more broadly in classical NHEJ. While both polymerases can utilize complementary DSB substrates with paired primer termini, Pol $\mu$ is uniquely capable of bridging broken DNA ends lacking break site microhomology ${ }^{11,12}$. Though there exists a plethora of biochemical $^{13-16}$ and structural ${ }^{17-21}$ information illustrating the activity of these Family X polymerases on single-strand break (SSB) substrates, understanding how each individual polymerase copes with DSB end-bridging is unclear. Thus far, only DSBbound crystal structures of murine $\mathrm{TdT}^{22,23}$ and a chimeric construct of murine TdT containing the Loop1 region from murine Pol $\mu^{24}$ are currently available, providing an important yet incomplete portrait of Family X polymerase behavior during NHEJ. We therefore present high-resolution crystal structures of the human Pol $\mu$ catalytic domain simultaneously engaging both ends of a complementary DSB substrate. A catalytically-poised pre-catalytic quaternary complex was trapped using a correctly paired nonhydrolyzable nucleotide, which could be exchanged with a hydrolyzable nucleotide to generate "snapshots" of the incorporation reaction proceeding in crystallo. These structures indicate that Pol $\mu$ provides a rigid scaffold, which can a

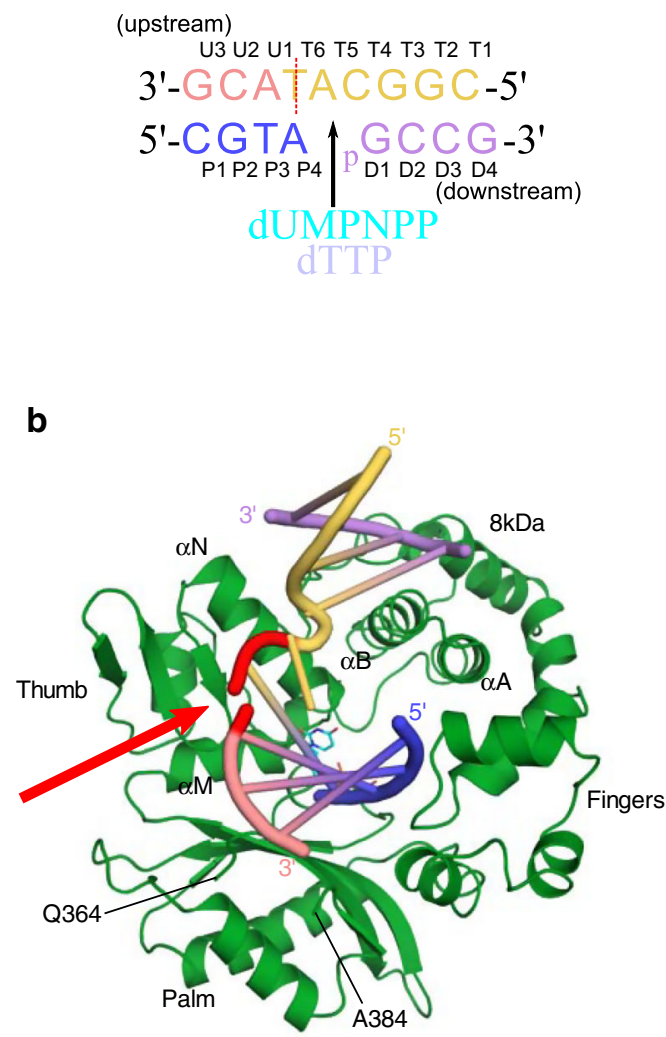

c

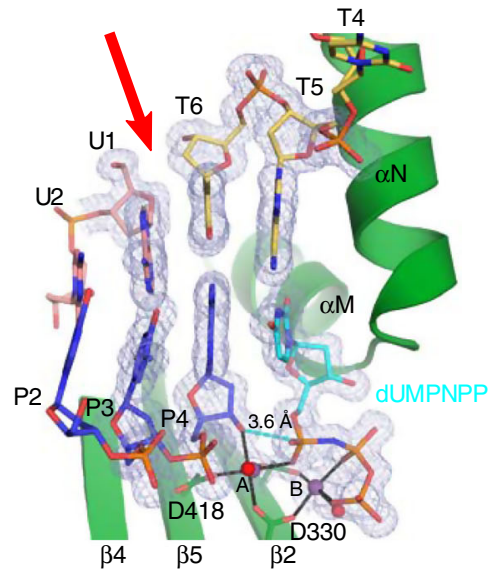

d

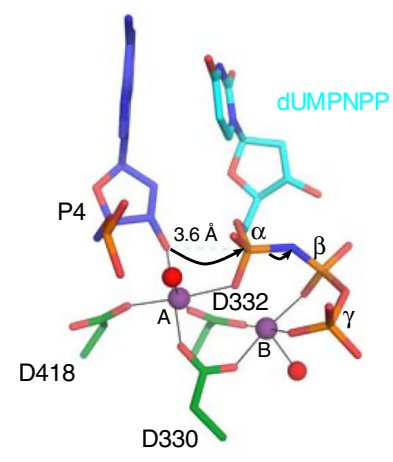

Fig. 1 Structural characterization of $\mathbf{h P o l} \boldsymbol{\mu} \Delta \mathbf{2}$ engaging a complementary DSB substrate. a DNA substrate crystallized with $\mathrm{hPol} \mu \Delta 2$ and nonhydrolyzable dUMPNPP. Template strand discontinuity is marked (dashed red line). b Pre-catalytic quaternary DSB (protein, dark green; DNA and dUMPNPP colored as in a; template break site, red) The ordered ends of Loop1 are indicated (Gln364/Ala384). c hPol $\mu \Delta 2$ pre-catalytic quaternary complex active site. $2 F_{o}-F_{c}$ electron density (gray mesh) contoured at $1 \sigma$. lonic interactions $\left(\mathrm{Mg}^{2+}\right.$ ion, purple; water molecules, red) are indicated by black bars. Interatomic distance between $3^{\prime}-\mathrm{OH}$ and $\alpha$-phosphate (dashed cyan line) was measured in PyMOL (Schrödinger). $\mathbf{d}$ Zoomed-in view of the hPol $\mu \Delta 2$ active center, with arrows indicating putative electron movements during reaction progression. 
Table 1 Data collection and refinement statistics.

\begin{tabular}{|c|c|c|c|}
\hline & $\begin{array}{l}\text { Pre-catalytic } \\
\text { complex }^{a, b}\end{array}$ & $\begin{array}{l}\text { Incomplete } \\
\text { incorporation }^{a} \text {, } \\
\text { b }\end{array}$ & $\begin{array}{l}\text { Post- } \\
\text { catalytic } \\
\text { complex }^{a, b}\end{array}$ \\
\hline & \multicolumn{2}{|c|}{ Data collection } & 6WIE \\
\hline $\begin{array}{l}\text { Space group } \\
\text { Cell dimensions }\end{array}$ & \multicolumn{3}{|c|}{ Cell dimensions } \\
\hline$a, b, c(\AA)$ & $\begin{array}{l}60.13 \\
62.23,118.05\end{array}$ & $\begin{array}{l}\text { 60.19, } \\
62.04,118.32\end{array}$ & $\begin{array}{l}60.07 \\
62.25,118.32\end{array}$ \\
\hline $\mathrm{A}, \beta, \gamma\left(^{\circ}\right)$ & $90,90,90$ & $90,90,90$ & $90,90,90$ \\
\hline Resolution $(\AA)$ & $\begin{array}{l}50-1.55 \\
(1.58-1.55)^{c}\end{array}$ & $\begin{array}{l}50-1.50 \\
(1.53-1.50)\end{array}$ & $\begin{array}{l}50-1.50 \\
(1.53-1.50)\end{array}$ \\
\hline$R_{\text {sym }}(\%)$ & $6.2(53.5)$ & $8.3(54.8)$ & $10.6(57.5)$ \\
\hline$|/ \sigma|$ & $26.63(1.5)$ & $28.47(2.24)$ & $18.54(1.77)$ \\
\hline Completeness (\%) & $99.5(98.1)$ & 99.8 (99.9) & $99.8(99.8)$ \\
\hline \multicolumn{4}{|l|}{ Refinement } \\
\hline Resolution $(\AA)$ & $35-1.55$ & $31.0-1.50$ & $35.0-1.50$ \\
\hline No. reflections & 64,729 & 71,176 & 71,906 \\
\hline$R_{\text {work }} / R_{\text {free }}(\%)$ & $16.55 / 17.94$ & $15.70 / 16.95$ & $16.99 / 18.68$ \\
\hline \multicolumn{4}{|l|}{ No. of atoms } \\
\hline Protein & 2563 & 2562 & 2564 \\
\hline DNA & 379 & $420^{d}$ & 399 \\
\hline Nucleotide & $28^{e}$ & $28^{e} / 9^{f}$ & 98 \\
\hline Water & 347 & 348 & 346 \\
\hline \multicolumn{4}{|l|}{ B-factors } \\
\hline Protein & 22.89 & 18.38 & 20.17 \\
\hline DNA & 19.06 & $13.87^{d}$ & 15.45 \\
\hline Nucleotide & $14.20^{\mathrm{e}}$ & $9.21^{e} / 14.56^{f}$ & $25.08 \mathrm{~g}$ \\
\hline Water & 33.38 & 31.09 & 31.04 \\
\hline \multicolumn{4}{|l|}{ R.m.s. deviations } \\
\hline Bond lengths $(\AA)$ & 0.009 & 0.009 & 0.009 \\
\hline Bond angles $\left({ }^{\circ}\right)$ & 1.038 & 1.087 & 1.030 \\
\hline \multicolumn{4}{|c|}{$\begin{array}{l}\text { aA single crystal was used to collect each data set. } \\
\text { bThese crystals were collected on the Southeast Regional Collaborative Access Team (SER- } \\
\text { CAT) 22-ID beamline at the Advanced Photon Source at Argonne National Laboratory. } \\
\text { cValues in parentheses are for highest-resolution shell. } \\
\text { Includes atoms from unincorporated and incorporated alternate conformations of residues P4 } \\
\text { and P5. } \\
\text { eNonhydrolyzable incoming dUMPNPP nucleotide. } \\
\text { fInorganic pyrophosphate leaving group. } \\
\text { gPartially disordered inorganic pyrophosphate leaving group. }\end{array}$} \\
\hline
\end{tabular}

accommodate both SSBs and complementary DSBs in a nearly indistinguishable manner.

\section{Results}

Pre-catalytic hPol $\mu \Delta 2$ quaternary complex with complementary DSB. A variant of the Pol $\mu$ catalytic domain with increased crystallizability and biochemical characteristics equivalent to those of the wildtype enzyme was used for this study (henceforth, referred to as $\mathrm{hPol} \mu \Delta 2^{21}$ ). In this variant, the flexible, non-conserved loop (Loop2) between $\beta$-strands 4 and 5 of the palm subdomain was deleted ( $\triangle$ Pro398-Pro410), and the ends of the $\beta$-strands were fused by a single glycine residue (Gly410). The $\mathrm{hPol} \mu \Delta 2$ pre-catalytic quaternary complex was incrementally assembled by first incubating the protein with an annealed downstream DNA duplex containing a $5^{\prime}$-phosphorylated downstream primer (Fig. 1a) to dictate correct binding, as Pol $\mu$ prefers to orient the nascent base pair binding site using the $5^{\prime}$ end of the gap rather than the $3^{\prime}$-end ${ }^{20}$, and its activity is stimulated by the presence of a phosphate at that position ${ }^{15}$. Synapsis is then achieved by the polymerase after addition of annealed upstream DNA-facilitated by $3^{\prime}$-primer terminal single-nucleotide complementarity (A:T), consistent with reports that Pol $\mu$ efficiently mediates end-bridging without other NHEJbinding partners ${ }^{25}$. Binding a correctly paired nonhydrolyzable

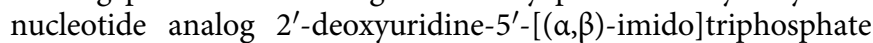
(dUMPNPP) in the active site leaves the enzyme poised for catalysis (PDB ID code 6WIC, Fig. 1b and Table 1). The upstream and downstream duplex regions are positioned distally from one another, an arrangement induced by an $\sim 90^{\circ}$ bend in the template backbone immediately downstream of the nascent base pair binding site. The upstream primer terminal sugar (residue P4) and that of the incoming dUMPNPP are observed with $\mathrm{C}^{\prime}$-endo sugar puckers, which leaves the $3^{\prime}-\mathrm{OH}$ ideally positioned for inline attack on the $\alpha$-phosphate (3.6 $\AA$ ) of dUMPNPP (Fig. 1c, d). Both divalent metal sites are occupied by $\mathrm{Mg}^{2+}$ ions, which are observed with octahedral coordination mediated by the $3^{\prime}-\mathrm{OH}$, Asp330, Asp332, Asp418, the triphosphate oxygens, and associated water molecules.

Protein-DNA interactions in the $\mathrm{hPol} \mu \Delta 2$ quaternary complex. From $h$ Pol $\mu \Delta 2$ 's perspective, the process of binding the downstream duplex to the $8 \mathrm{kDa}$ subdomain likely occurs in a similar fashion, regardless of whether the DNA substrate contains an intact or discontinuous template strand, as relevant breaks occur upstream of the nascent base pair binding site. The $\sim 90^{\circ}$ bend in the template backbone between residues T4 and T5 opens the helix to allow access of incoming nucleotides to the nascent base pair binding site, positioning the $5^{\prime}$-phosphate on the downstream primer $>20 \AA$ from that location (Fig. 2a). This bend is reinforced by multiple putative hydrogen bonding interactions from the nonbridging phosphate oxygens of residues T4-T6 to the sidechains of Arg442, Arg449 (partially disordered), and Lys450 (Table 2). The position of the downstream duplex is stabilized by van der Waals interactions with the backbone of Gly174 on the N-terminal end of $\alpha$-helix B, anchoring the $5^{\prime}$ phosphate to the $8 \mathrm{kDa}$ subdomain via putative hydrogen bonds with the sidechains of Arg175 (partially disordered) and His208 (Fig. 2a and Table 2). Interestingly, mutations of Gly174 and Arg175, which diminish the fidelity and efficiency of Pol $\mu$ 's activity in $\mathrm{NHE}^{26}$, have been discovered in skin and ovarian cancers, respectively 27,28 .

Electron density for the upstream template strand clearly highlights the backbone discontinuity between residues T6 and U1 (Fig. 1c). Both sides of the break in the pre-catalytic complex are stabilized by a hydrogen bonding network within Polp's substrate binding cleft (Fig. $2 \mathrm{~b}$ and Table 2). The $\mathrm{O}^{\prime}$ atom on upstream template residue $\mathrm{U} 1$ is solvent-exposed, but the $\mathrm{O}^{\prime}$ atom on the $3^{\prime}$ end of downstream template residue T6 orients toward the protein surface and putatively hydrogen bonds with Asn457. Arg445 also lies in the minor groove within hydrogen bonding distance of the T6 base. Arg387 interacts with the U1 base upstream of the break. The U2-U3 phosphate lies within hydrogen bonding distance of the Glu386 and Arg387 backbone amide nitrogens, whereas Gln364 putatively interacts with the $3^{\prime}-\mathrm{OH}$ of residue U3.

Upstream primer strand positioning is stabilized by van der Waals, ionic, and hydrogen bonding interactions (Fig. $2 \mathrm{c}$ and Table 2). The aromatic sidechains of Trp434 and Phe389 likely stabilize the sugar moieties of the primer terminal and penultimate nucleotides, respectively, via $\pi-\mathrm{CH}$ interactions. The upstream P1-P2 phosphate is secured by multiple interactions between its nonbridging oxygens and the Thr250 sidechain, its backbone amide, and the backbone amide of Gly247. The $\mathrm{P} 2-\mathrm{P} 3$ phosphate is stabilized by a putative hydrogen bond with the backbone amide of Gly245 and an interaction with the $\mathrm{Na}^{+}$ coordinated by the $\mathrm{HhH} 2$ (helix-hairpin-helix) motif (residues Thr241-Val246) that is conserved throughout the Family X polymerases ${ }^{6}$. The $\mathrm{P} 3-\mathrm{P} 4$ phosphate is tethered near the catalytic 


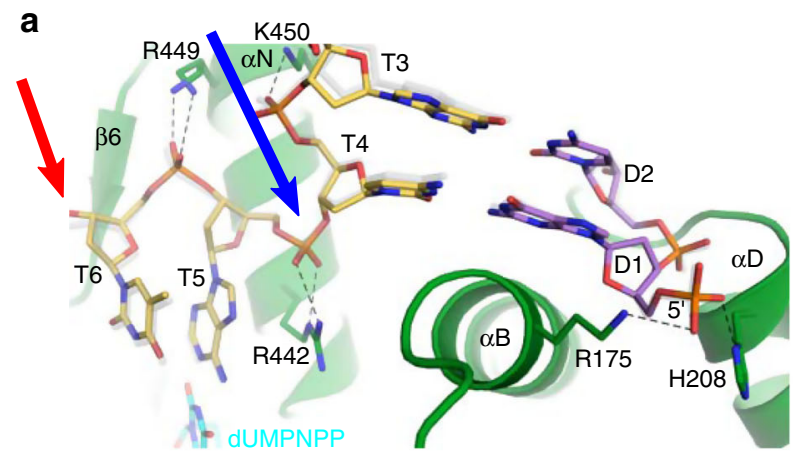

b

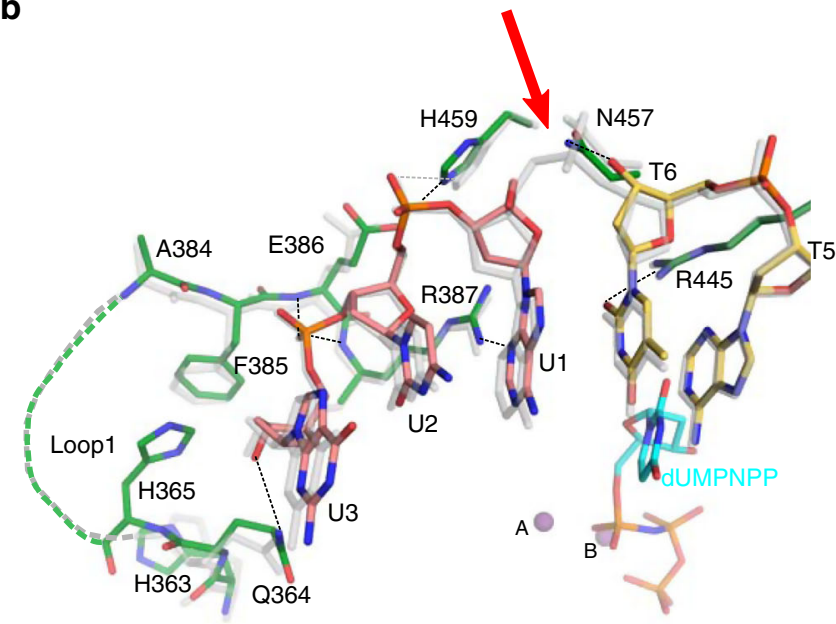

C

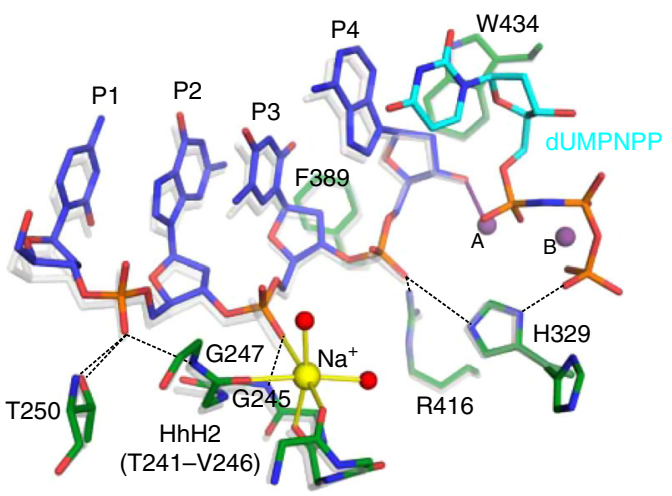

Fig. 2 Comparison of $\mathrm{hPol} \mu \Delta \mathbf{2}$ interactions with SSB or complementary DSB substrates. Superposition of $h \mathrm{Pol} \mu \Delta 2$ pre-catalytic complexes bound to a DSB (colored as in 1a) or a 1nt-gapped SSB (gray, PDB ID code $4 \mathrm{MO4}^{21}$ ). Interactions involving downstream duplex $\mathbf{a}$, template strand $\mathbf{b}$ and upstream primer strand $\mathbf{c}$ near the break site are diagrammed (Table 2). Putative hydrogen bonds in DSB and SSB complexes drawn as dashed lines (black or gray, respectively). lonic interactions (solid lines) are color-coordinated with metal identity. Red and blue arrows indicate template backbone break and bend, respectively. Arg449 and

Arg175 sidechains in a are partially disordered and only the ordered regions are included in the model.

center via a hydrogen bond with Arg416, which has been shown to be dispensable for nucleotide incorporation on SSBs but essential for DSB repair ${ }^{29}$. The His329 sidechain, thought to bridge the primer terminal phosphate and incoming nucleotide during $\mathrm{NHEJ}^{19}$, is observed in multiple conformations, one of which lies within long-range hydrogen bonding distance of a $\mathrm{P} 3-\mathrm{P} 4$ phosphate oxygen. Correct positioning of the primer

\begin{tabular}{|c|c|c|}
\hline Region & Interacting atoms & $\begin{array}{l}\text { Interatomic } \\
\text { distance }^{\mathrm{a}}(\AA)\end{array}$ \\
\hline \multirow{7}{*}{$\begin{array}{l}\text { Downstream } \\
\text { primer strand }\end{array}$} & Arg175b NE-D1 OP1 & 3.3 \\
\hline & His208 ND1-D1 OP2 & 2.6 \\
\hline & Ser209 N-D2 OP1 & 3.0 \\
\hline & Gly206 N-D2 OP1 & 3.0 \\
\hline & His208 N-D2 OP2 & 3.0 \\
\hline & Glu207 N-D2 OP2 & 3.3 \\
\hline & His204 N-D3 OP1 & 2.9 \\
\hline \multirow[t]{6}{*}{$\begin{array}{l}\text { Downstream } \\
\text { template strand }\end{array}$} & $\begin{array}{l}\text { Arg 449b NE -- T6 OP1/ } \\
2\end{array}$ & $2.6-3.3$ \\
\hline & Arg442 NE-T5 OP2 & 2.9 \\
\hline & Arg442 NH2-T5 OP1 & 3.0 \\
\hline & Ly450 NZ-T4 OP1 & 2.6 \\
\hline & Asn457 ND2-T6 O3' & 2.9 \\
\hline & Arg445 NH1-T6 O2 & 2.9 \\
\hline Upstream & Arg387 NH1-U1 N3 & 3.0 \\
\hline \multirow[t]{3}{*}{ template strand } & Glu386 N-U3 OP1 & 2.8 \\
\hline & Arg387 N-U3 OP1 & 3.0 \\
\hline & Glu364 NE2-U3 O3' & 3.1 \\
\hline Upstream & Thr250 N-P2 OP1 & 3.1 \\
\hline \multirow{6}{*}{ primer strand } & Thr250 OG1-P2 OP1 & 2.7 \\
\hline & Gly247 N-P2 OP1 & 2.9 \\
\hline & Gly245 N-P3 OP1 & 2.9 \\
\hline & Arg416 NH2-P4 OP1 & 2.8 \\
\hline & His329 & 3.6 \\
\hline & NE2 (A)-P4 OP1 & \\
\hline
\end{tabular}

terminal $3^{\prime}-\mathrm{OH}$ is crucial for catalysis and is largely mediated by coordination with the catalytic $\mathrm{Mg}^{2+}$.

The observed $h P o l \mu \Delta 2$ conformation is catalytically competent. Transferring the $h \operatorname{Pol} \mu \Delta 2$ pre-catalytic quaternary complex crystals from a cryoprotectant solution containing nonhydrolyzable dUMPNPP to a cryoprotectant solution containing hydrolyzable dTTP allows nucleotide exchange and insertion in crystallo, yielding a structure exhibiting incomplete ( 40\%, PDB ID code 6WID, Table 1 and Fig. 3a-b) or nearly complete (PDB ID code 6WIE, Table 1 and Fig. 3c) incorporation after a longer soak. Successful nucleotide incorporation within the crystalline lattice indicates that the observed pre-catalytic quaternary conformation is indeed catalytically competent. Superposition of pre- and fully post-catalytic complexes reveals no large-scale movements of protein subdomains, DNA substrate, or active site residues during nucleotide insertion ( $0.084 \AA$ RMSD over $278 \mathrm{Ca}$ atoms, Fig. 3d). There is a slight (1.4 $\AA$ ) shift of the $3^{\prime}$-primer terminus toward the a-phosphate of the newly incorporated nucleotide, allowing phosphodiester bond formation. A correlated adjustment $(\leq 1.1 \AA)$ of the Trp434 sidechain is also observed, mediated by a rotation around the CB-CG bond (Fig. 3e). These subtle motions appear to be a normal consequence of nucleotide insertion by Pol $\mu^{21}$ and are likely observed regardless of DNA substrate configuration. There is no observed density for a third "product metal" in these structures (Fig. 3), even in the structure exhibiting incomplete incorporation where the "product metal" might be expected, which is consistent with previously published reports ${ }^{30}$. Upon reaction completion, the pyrophosphate leaving group becomes partially disordered and replaced by solvent molecules, which cannot be definitively modeled. 
a
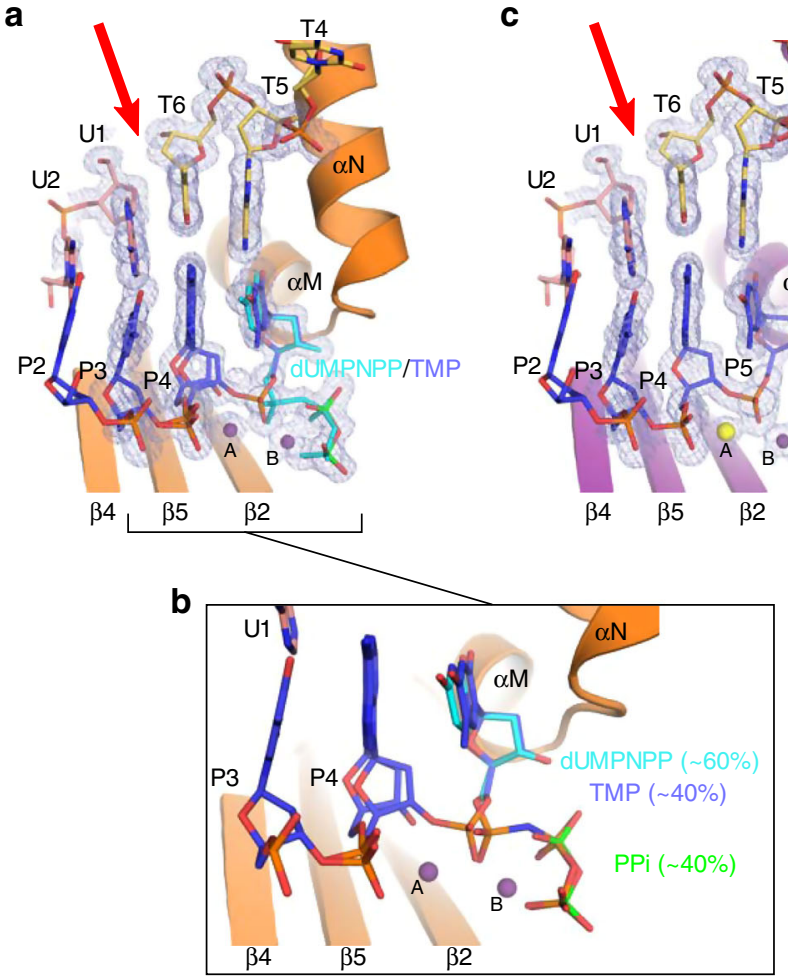

c

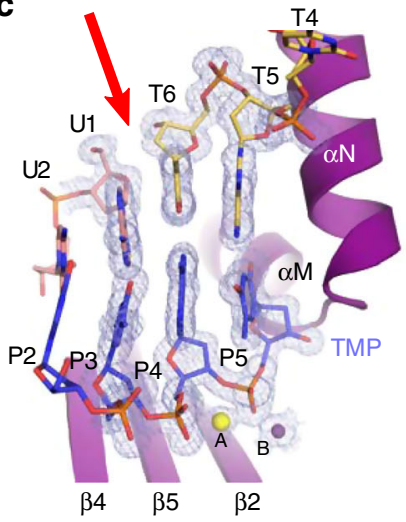

d

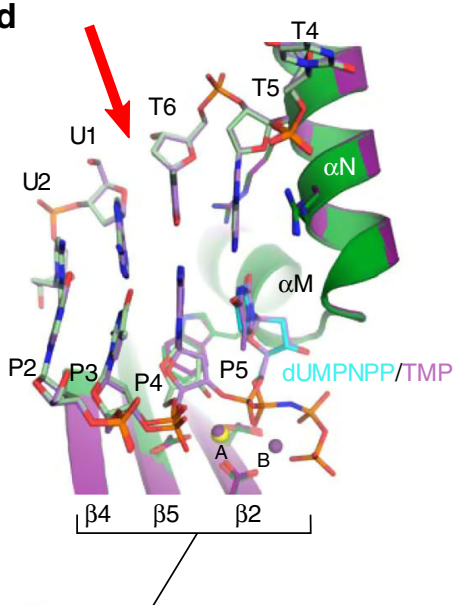

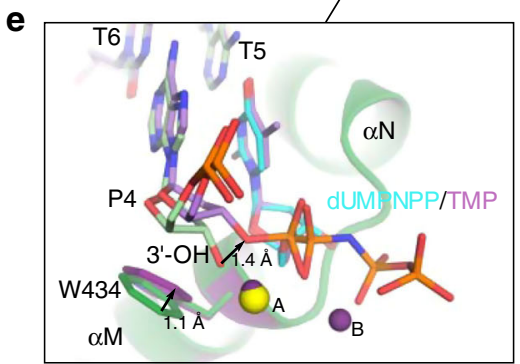

Fig. 3 Catalytically competent $\mathbf{h P o l} \boldsymbol{\mu} \Delta \mathbf{2}$ active site geometry leads to nucleotide incorporation in crystallo. a Structural snapshot of the $\mathrm{hPol} \mu \Delta 2$ active site, exhibiting incomplete incorporation of the hydrolyzable dTTP nucleotide, with a zoomed view $\mathbf{b}$ of the primer terminal and unincorporated dUMPNPP (cyan) or newly incorporated TMP nucleotides. Protein components are shown as an orange ribbon; DNA is drawn in stick (colored as in Fig. 1a-b). Mg2+ ions are shown as purple spheres. The pyrophosphate ( $\mathrm{PPi}$ ) leaving group is drawn in green. c Fully post-catalytic nicked complex. Though $\mathrm{Mg}^{2+}$ fully occupies the $B$ site in the post-catalytic complex, the $A$ site metal has been partially replaced by $\mathrm{Na}^{+}$(yellow). $2 F_{0}-F_{c}$ electron density for active site components is shown as a gray mesh (contoured at $1 \sigma$ ). Red arrow indicates template backbone break. Superposition of hPol $\mu \Delta 2$ pre- (green; dUMPNPP in cyan) and post-catalytic nicked (purple; $\mathrm{Na}^{+}$ion in yellow) complexes, shown as global $\mathbf{d}$ or zoomed in e views. Positional shifts are shown by black arrows, with the measured distances indicated.

5'-phosphate binding becomes critical on tenuous DNA substrates. Previous studies have shown that interactions with or near the $5^{\prime}$-phosphate on the downstream duplex can have deleterious consequences for efficiency and fidelity of end-joining activity by Pol $\mu^{26,31}$. Disease-associated mutations of Gly174 and Arg175 demonstrated only subtle differences in polymerization efficiency on a single-nucleotide SSB substrate, which became more pronounced as the SSB became a complementary DSB. Repair of a noncomplementary DSB, which lacks microhomology at the break site to facilitate synapsis, was abrogated further ${ }^{26}$. Moreover, substitution of Arg175 with histidine showed lessdeleterious effects on end-joining than did replacement with alanine $^{31}$. In order to determine whether His208 (Fig. 2a) binding to the $5^{\prime}$-phosphate is similarly required for contribution of Pol $\mu$ to end-joining, we generated an alanine substitution at this position and assayed its ability to mediate single-nucleotide gapfilling on both partly complementary and noncomplementary DSB ends. Similar to previous reports of the Gly174 and Arg175 mutants, the H208A mutant demonstrated decreased end-joining efficiency on complementary DSB ends (Fig. 4, top), compared with the wildtype enzyme. Activity of this mutant was negligible when compared with a no-polymerase control when using noncomplementary overhangs (Fig. 4, bottom).

\section{Discussion}

Superposition of the human $h \operatorname{Pol} \mu \Delta 2$ pre-catalytic quaternary complementary DSB and either the human (Fig. 5a, b and Fig. 2) or mouse (Fig. 5c, d) Pol $\mu$ ternary SSB complexes reveal a high degree of global similarity ( $0.34 \AA$ RMSD over $284 \mathrm{C} \alpha$ atoms and
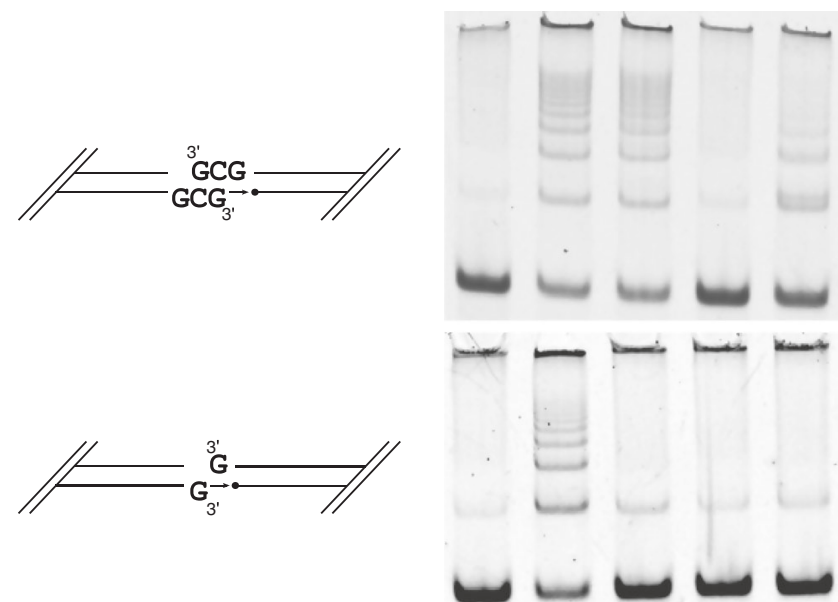

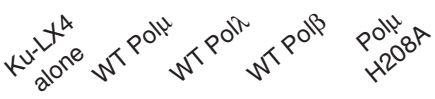

Fig. 4 Qualitative assessment of the role of His208 in NHEJ proficiency. Activity of full-length wildtype hPol $\mu$ versus the Pol $\mu \mathrm{H} 208 \mathrm{~A}$ mutant in in vitro NHEJ concatamerization assay using complementary (top) or noncomplementary (bottom) DSB ends, as depicted in cartoons beside each panel. Downstream primer is $5^{\prime}$-phosphorylated (black circle). Pols $\lambda$ and $\beta$ are included for reference, as Pol $\beta$ shows no detectable activity for either substrate, whereas Pol $\lambda$ is active only on the partly complementary DSB. Source data are provided as a Source Data file. 
a

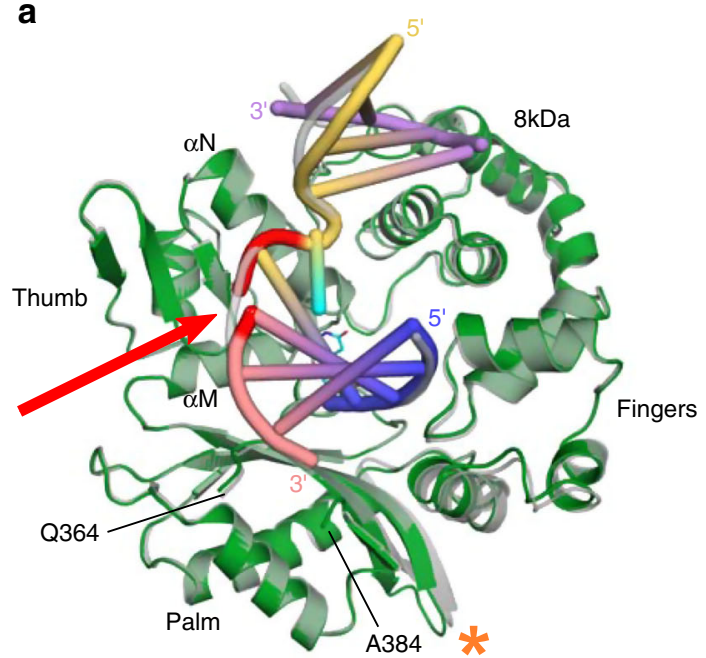

C

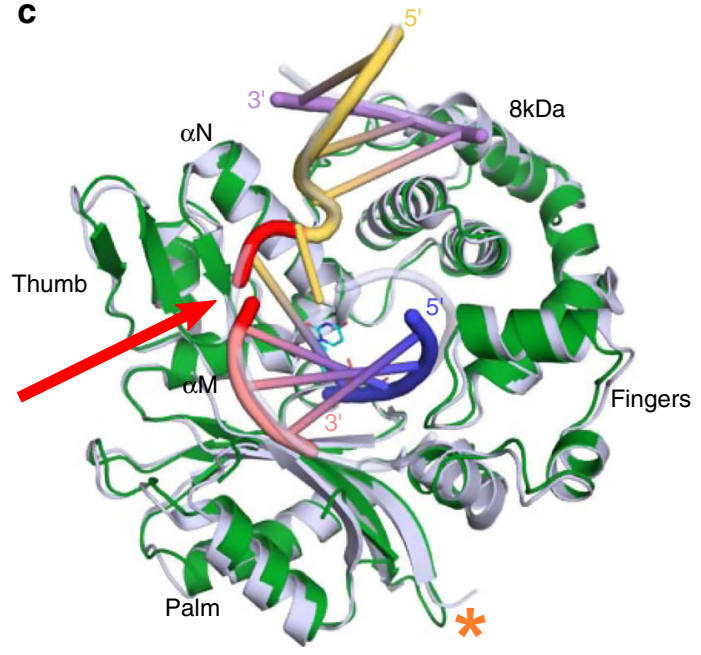

b

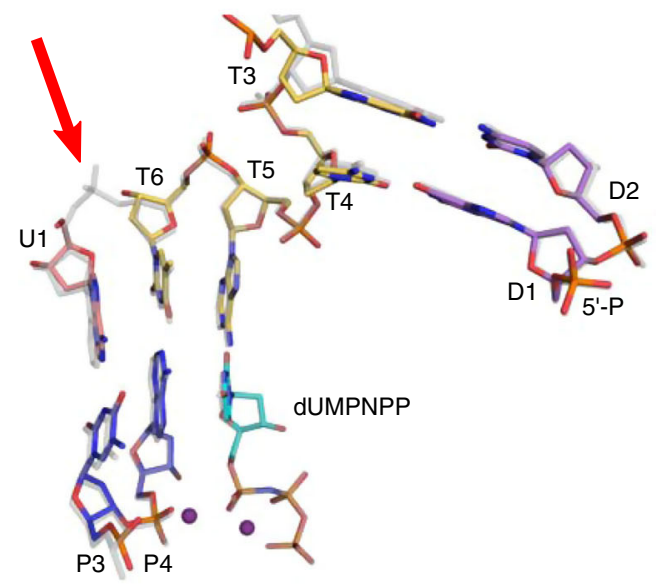

d

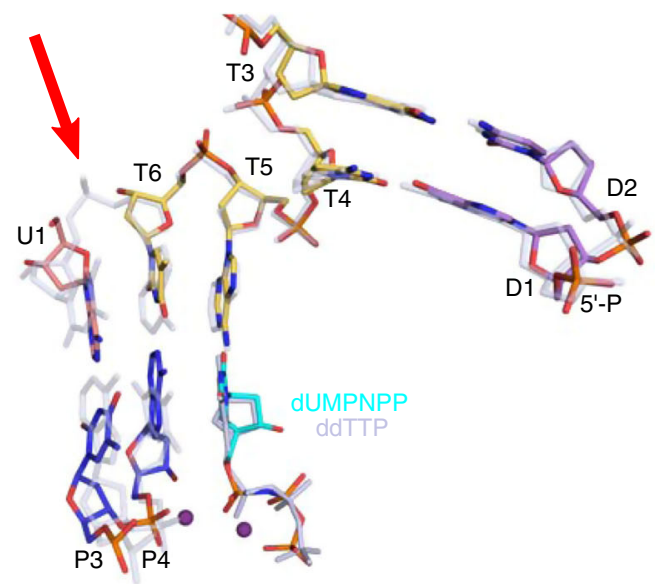

Fig. 5 Comparison of the $\mathbf{h P o l} \boldsymbol{\mu} \mathbf{\Delta} \mathbf{2}$ complementary DSB and other human or mouse Pol $\boldsymbol{\mu}$ crystal structures. Superposition of $h$ Pol $\mu \Delta 2$ complementary DSB (colored as in Fig. 1a-b) with a hPol $\mu \Delta 2$ (gray, PDB ID code $4 \mathrm{MO4}^{21}$ ) or $\mathbf{c}$ mouse Pol $\mu$ (light blue, PDB ID code 2IHM ${ }^{19}$ ) 1nt-gapped SSB substrates, with zoomed-in view of superimposed human $\mathbf{b}$ or mouse $\mathbf{d}$ DNA substrates in stick. Red arrow and orange asterisk indicate the location of the broken template backbone and the $\mathrm{hPol} \mu \Delta 2$ Loop2 deletion, respectively.

0.81 Å over $287 \mathrm{Ca}$ atoms, respectively). Subtle differences can be observed in the position of the Loop2 region (disordered in mouse Pol $\mu$ and deleted $\Delta$ Pro389-Pro410 in the human Pol $\mu$ ) and at the distal ends of the DNA duplex, all of which can be influenced by crystal packing and DNA pseudo-stacking between the crystal forms. Interactions between protein and either the $5^{\prime}$ phosphate (Fig. 2a) or upstream primer strand (Fig. 2c) are conserved between both substrate types in these structures, but slight variations are observed in residues surrounding the break in the human structures, namely the sidechain conformations of Asn457 and His459 (Fig. 2b). His459 lies within long-range hydrogen bonding distance of the T7-T8 phosphate oxygens in the SSB $(3.3 \AA)$, but likely interacts instead with Glu386 (2.7 ̊̊) on the end of $\beta$-strand 4 near Loop1 in the DSB complex. The precise nature of these interactions and their contributions to catalysis by Pol $\mu$ in SSB and DSB repair are currently unclear, as previous studies have shown that glycine substitution of His459 had no apparent effect on single-nucleotide gap-filling on either SSB or DSB substrates, and the N457D mutant was profoundly impaired on all substrates ${ }^{32}$. The similarities between the singleand DSB complexes therefore suggest that Pol $\mu$ addresses DSB substrates containing break site complementarity in a manner analogous to its engagement of gapped SSB substrates, and that the polymerization reaction proceeds through the same mechanism.

Comparison of the $\mathrm{hPol} \mu \Delta 2$ quaternary complementary DSB structure with those available for mouse $\mathrm{TdT}$ reveals distinct differences. Superposition of the $\mathrm{hPol} \mu \Delta 2$ complementary DSB with that of mouse TdT bound to a DSB substrate of similar configuration (PDB ID code 5D46 ${ }^{23}$ ) shows structural correlation for the protein (RMSD of $1.04 \AA$ over $275 \mathrm{Ca}$ atoms, Fig. 6a), but less for the DNA (Fig. 6b). Equivalent residues comprising the primer terminus/nascent base pair binding site (referred to as an A-form "mini-helix" in an otherwise B-form duplex), and the downstream duplex show very similar positioning, but duplex upstream of the primer terminal base pair ( $\mathrm{P} 4: \mathrm{T} 6$ in $\mathrm{hPol} \mu \Delta 2)$ is "wedged" open in mouse TdT by insertion of Loop1 residues into the duplex. A similar phenomenon is observed in another mouse TdT DSB synaptic complex mediated by single-nucleotide complementarity on the template strand (PDB ID code $4 \mathrm{QZZ} 8^{22}$, RMSD of $1.1 \AA$ over $280 \mathrm{Ca}$ atoms, Fig. 6c, d). The upstream primer strand in this structure is also "wedged" open, in the absence of an upstream template strand, suggesting that this behavior occurs independently of upstream base pairing in $\operatorname{TdT}$ (Fig. 6e). The "wedging" phenomenon is not observed in the $\mathrm{hPol} \mu \Delta 2$ complementary DSB substrate. 
a

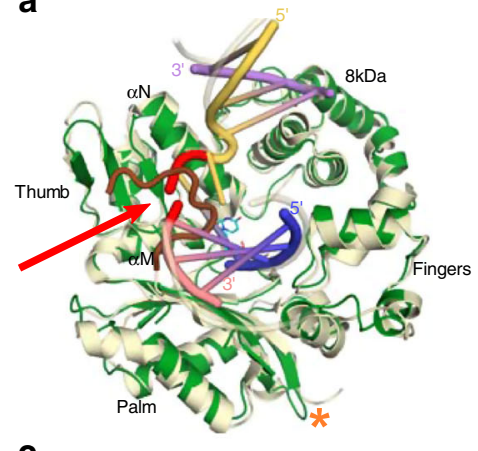

C

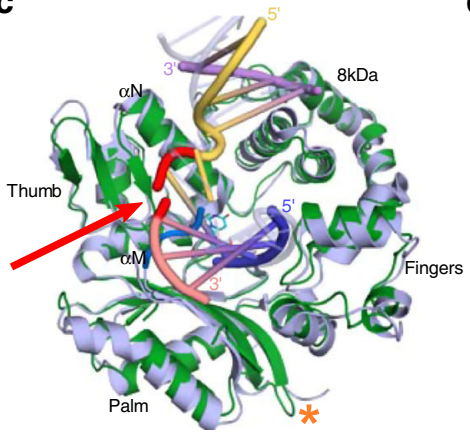

b

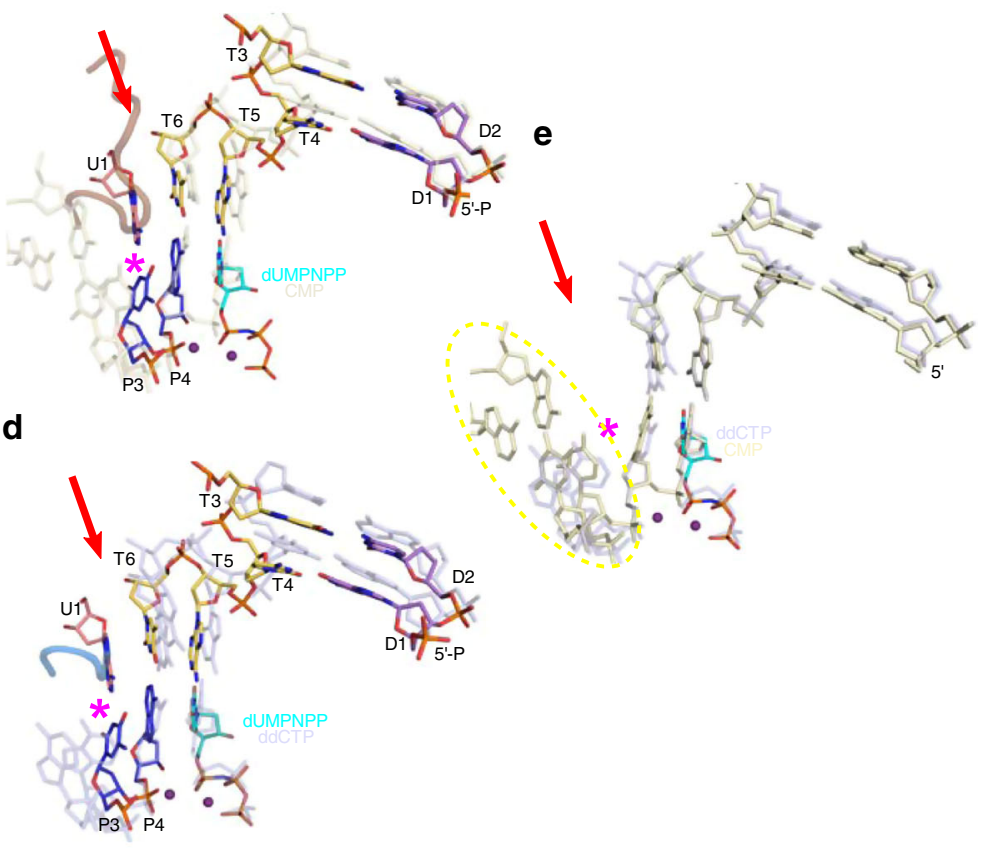

Fig. 6 Comparison of the $\mathbf{h P o l} \boldsymbol{\mu} \boldsymbol{\Delta} \mathbf{2}$ complementary DSB and mouse TdT crystal structures. Superposition of hPol $\mu \Delta 2$ complementary DSB (colored as in Fig. 1a-b) with mouse TdT DSB synapse with a nicked complementary DSB substrate (khaki, PDB ID code 5D4623) or a c single-nucleotide complementary DSB substrate otherwise lacking an upstream template (light blue, PDB ID code 4QZ822), with zoomed-in view of superimposed DNA substrates $\mathbf{b}$, $\mathbf{d}$. Loop1 residues from TdT are drawn in cartoon (brown and blue, respectively). e Superimposed DNA substrates from the TdT crystal structures, colored as in c, $\mathbf{d}$ to highlight the "wedged" upstream residues (dashed yellow circle). Red arrow indicates the location of the broken template strand. The magenta and orange asterisks indicate the positions of the "wedged" upstream residues and the hPol $\mu \Delta 2$ Loop2 deletion, respectively.

A recent study of a chimeric form of mouse TdT harboring the Loop1 region from mouse Pol $\mu$ (henceforth, referred to as TdT- $\mu$ ) included crystal structures of the chimera in complex with a 1ntgapped SSB substrate (PDB ID code $6 \mathrm{GO}^{24}$, Fig. $7 \mathrm{a}, \mathrm{b}$ ) and with a noncomplementary DSB synaptic complex reinforced by a triplex-forming oligonucleotide (PDB ID code $6 \mathrm{GO}^{24}$, Fig. $7 \mathrm{c}$, d). Superposition of the $\mathrm{hPol} \mu \Delta 2$ complementary DSB synaptic complex with these structures yielded some intriguing differences. As with the superpositions with $\mathrm{TdT}$, comparisons of the TdT- $\mu$ chimeric complexes showed similarities in global protein structure (1.02-1.03 $\AA$ over 274-276 Ca atoms), but some differences in DNA structure. The TdT- $\mu$ crystal structure with the 1ntgapped SSB shows strong similarity of the DNA substrate position with that of the $h P o l \mu \Delta 2$ complementary DSB, and the "wedged open" upstream duplex is not observed in either structure (Fig. $7 \mathrm{~b}$ ). In the TdT- $\mu$ noncomplementary DSB, however, the $3^{\prime}$-terminal base of the downstream template strand-which could mispair with the upstream primer terminal base, but is instead disordered-leaving an unpaired primer terminus (Fig. 7d, e). As in the crystal structures of TdT, the duplex upstream of the primer terminal nucleotide is observed in a "wedged" open conformation, though not by direct intervention of the chimeric mouse Pol $\mu$ Loop1 residues, as occurs in wildtype TdT. It is therefore tempting to speculate that Pol $\mu$ might utilize different modes of substrate binding, based on the sequence and structure configuration at the break site. Opening of the upstream duplex in a noncomplementary DSB could provide more access of the Loop1 region to interact with and stabilize the broken template strand, but may not be required for repair of complementary DSBs.

Among the Family $\mathrm{X}$ polymerases, the Loop1 motif is not required for repair of complementary DSB ends, but is critical for repair of DSBs lacking microhomology ${ }^{12}$. That Loop1 is disordered in these structures is perhaps unsurprising, given that repair of DSB substrates containing as little as one complementary base pair at the break site is Loop1-independent. Loop1 is largely disordered in all available structures of human or mouse Pol $\mu$-which contrasts with structures of TdT engaging a similar complementary DSB substrate, wherein an ordered Loop1 contributes to break site interactions (Fig. 6b) ${ }^{23}$. Comparison of the Loop1 conformations in the Pol $\mu$ structures, however, may provide some insight. Superposition of the $h P o l \mu \Delta 21 \mathrm{nt}$-gapped SSB and complementary DSB complexes shows that, although Loop1 is disordered in both structures, its overall trajectory and extent of disorder are similar (Fig. 2a and Fig. 8, green and purple dashed lines). In contrast, however, the overall trajectory of Loop1 in the human versus the mouse Pol $\mu$ SSB structures widely differ (Fig. 8, purple and blue dashed lines, respectively). Loop1 also displays different conformations in the human Pol $\mu$ complexes with different SSB substrates (1nt-gapped SSB, purple and 2nt-gapped SSB, orange). Although we cannot rule out the possibility that its conformations are influenced by crystal packing interactions, Loop1 likely adopts a conformation capable of stabilizing the template strand of any given substrate configuration, which becomes essential as the extent of break site complementarity decreases.

Since the Family X polymerases must contend with both ends of their substrates, often separated by $>20 \AA$ distance owing to the $\sim 90^{\circ}$ bend (Fig. $2 \mathrm{a}$ ) in the template backbone, strong interactions with the $5^{\prime}$-phosphate, the primer terminus, and template strands are required for correct catalytically-competent geometry. This theory is supported by a variety of mutagenesis experiments, wherein different substrate interactions have been removed and interrogated for their roles in subsequent repair. Alanine substitution mutagenesis of Arg416 $6^{29}$ and His $329^{19}$ are thought to hinder correct positioning of the primer strand for catalysis 
a

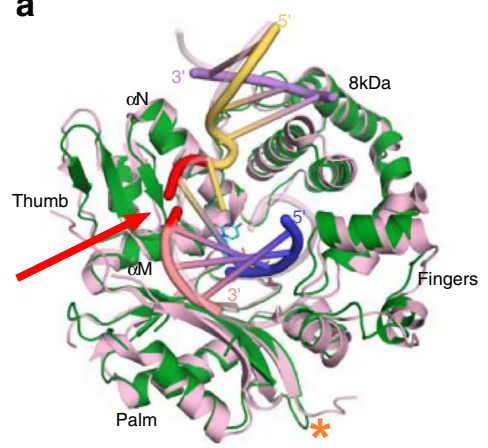

C

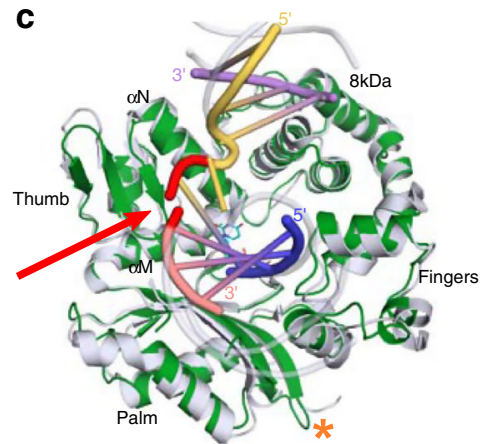

b

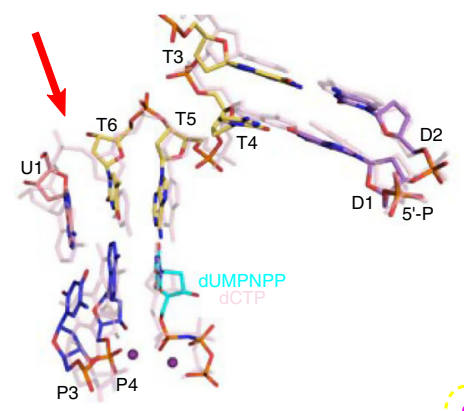

d

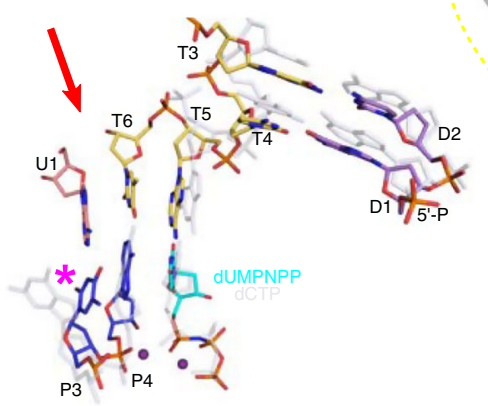

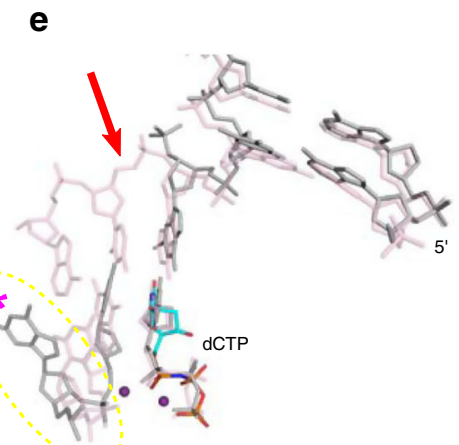

Fig. 7 Comparison of the $h P o l \mu \Delta \mathbf{2}$ complementary DSB and structures of the mouse TdT chimera harboring the Pol $\boldsymbol{\mu}$ Loop1 region. Superposition of $\mathrm{hPol} \mu \Delta 2$ complementary DSB (colored as in Fig. 1a-b) with mouse TdT a 1nt-gapped SSB (light pink, PDB ID code 6GO524) or c synapse with noncomplementary DSB (light gray, PDB ID code 6GO724) substrates, with zoomed-in view of superimposed DNA substrates b, d. e Superimposed DNA substrates from the chimeric TdT- $\mu$ crystal structures, colored as in c, d, to highlight the "wedged" upstream residues (dashed yellow circle). Red arrow indicates the location of the broken template strand. The magenta and orange asterisks indicate the positions of the "wedged" upstream residues and the $\mathrm{hPol} \mu \Delta 2$ Loop2 deletion, respectively.

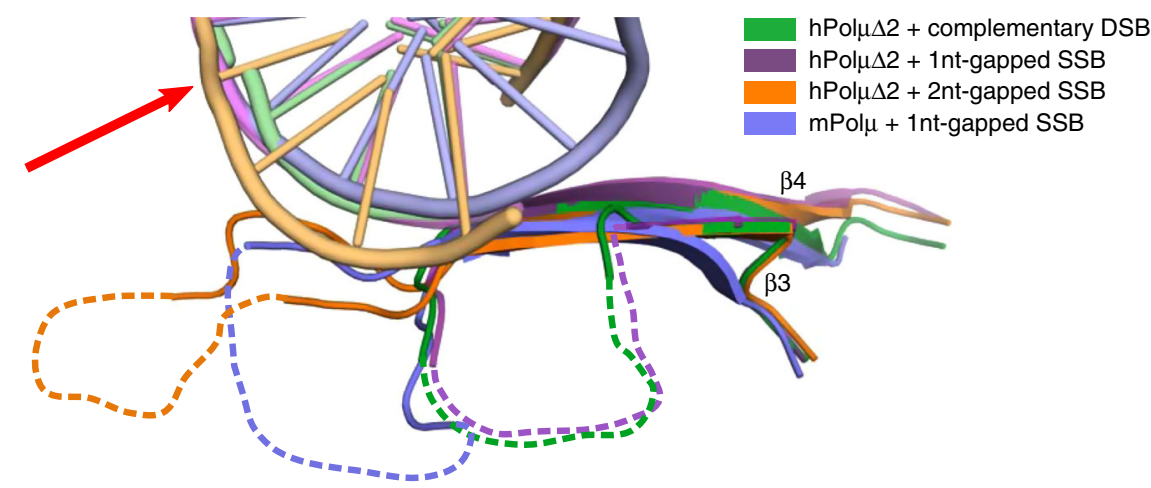

Fig. 8 Variability in Pol $\mu$ Loop1 conformations. Superpositions of mouse Pol $\mu$ SSB (protein in blue, DNA in light blue, PDB ID code $21 H M^{19}$ ), and human Pol $\mu \Delta 2$ SSB (1nt-gapped SSB with protein in purple, DNA in lavender, PDB ID code 4M0421; 2nt-gapped SSB in orange, DNA in light orange, PDB ID code $4 \mathrm{YD}^{20}$ ) with the $\mathrm{hPol} \mu \Delta 2$ complementary DSB structure (protein in green, DNA in light green, PDB ID code $6 \mathrm{WIC}$ ). $\beta$-strands 3 and 4 are drawn, with the ordered ends of Loop1 illustrating its projected trajectory. The disordered regions of Loop1 are hypothetically modeled by dashed lines in colors correlated with the protein structure. Red arrow indicates the location of the broken template strand in the complementary DSB.

(Fig. 2c). Likewise, loss of $5^{\prime}$-phosphate identification and positioning owing to mutations of Gly174 ${ }^{26}$, Arg $175^{31}$, or His208 (Fig. 4) lead to diminished repair capacity. These mutations have increasingly profound effects as the complexity of the repair substrate increases-SSB $<$ complementary $\mathrm{DSB}<$ noncomplementary DSB. These results suggest that substrate binding by $\mathrm{Pol} \mu$ is multifactorial, with myriad interactions working in synergy. In more simplistic DNA substrate configurations, interactions in each key area (primer strand, 5' -phosphate, etc) serve a redundant role for the others. However, as synapsis becomes more tenuous, each individual interaction becomes critical. The DSB-bound Pol $\mu$ structures presented in this study provide a greater understanding of how Pol $\mu$ addresses different substrate configurations, and how interactions with these substrates contribute to DSB repair in NHEJ.

\section{Methods}

Expression and purification of human Pol $\mu$ constructs. Full-length human Pol $\mu$ and a crystallization variant of the human Pol $\mu$ catalytic domain $(\mathrm{hPol} \mu \Delta 2$; Prol32Ala494 with Pro398-Pro410 of Loop2 deleted and replaced by Gly410) were cloned into the pGEXM vector ${ }^{21}$ and expressed in Rosetta2 (DE3) cells. $h P o l \mu \Delta 2$ was expressed in Rosetta2 (DE3) cells in LB medium supplemented with $100 \mu \mathrm{g} \mathrm{mL}^{-1}$ ampicillin and $35 \mu \mathrm{g} \mathrm{mL}-1$ chloramphenicol. Cells were grown at $37^{\circ} \mathrm{C}$ to an $\mathrm{OD}_{600 \mathrm{~nm}}$ of 0.8 , at which point the temperature was decreased to $18^{\circ} \mathrm{C}$ for $\sim 30$ minutes. Protein expression was induced by addition of isopropyl- $\beta$-D-thiogalactoside 
(IPTG) to a final concentration of $0.4 \mathrm{~mm}$, and continued overnight at $18^{\circ} \mathrm{C}$. The cells were pelleted and resuspended in lysis buffer $(25 \mathrm{~mm}$ Tris $\mathrm{pH} 8,500 \mathrm{~mm} \mathrm{NaCl}, 5 \%$ glycerol, $1 \mathrm{~mm}$ DTT) supplemented with $1 \mathrm{~mm}$ phenylmethylsulfonyl fluoride (PMSF) and cOmplete EDTA-free protease inhibitor tablets ( 1 tablet per $40 \mathrm{~mL}$ volume of lysis buffer). The cells were lysed by sonication and the lysate was subsequently cleared by centrifugation. Soluble protein was bound in-batch to glutathione $4 \mathrm{~B}-\mathrm{Sepharose}$ resin and subjected to on-resin TEV cleavage overnight at $4{ }^{\circ} \mathrm{C}$. Pol $\mu$ was then purified by size-exclusion chromatography on a Superdex200 26/600 column, equilibrated with lysis buffer. $h \operatorname{Pol} \mu \Delta 2$ was then further purified by ionexchange chromatography, using a Mono Q 5/50 GL column equilibrated in the final storage buffer specific for each construct. The purified protein did not bind the column and was observed in the flow-through. Purified full-length Pol $\mu$ was concentrated in $25 \mathrm{~mm}$ Tris $\mathrm{pH} 8,100 \mathrm{~mm} \mathrm{NaCl}, 5 \%$ glycerol, $1 \mathrm{~mm}$ DTT, and $10 \mathrm{~mm}$ $\mathrm{MgCl}_{2}$ buffer. The crystallization variant was concentrated in $25 \mathrm{~mm}$ Tris, $\mathrm{pH} 8,75$ $\mathrm{mm} \mathrm{NaCl}, 5 \%$ glycerol, and $1 \mathrm{~mm}$ DTT. Purified proteins were flash frozen in liquid nitrogen and stored at $-80^{\circ} \mathrm{C}$

\section{$\mathrm{hPol} \mu \Delta 2$ co-crystallization with DSB substrate and incoming dUMPNPP. The} following DNA oligonucleotides (Integrated DNA Technologies) were used to generate the complementary DNA DSB substrate: upstream template (5'-ACG- $\left.3^{\prime}\right)$, upstream primer $\left(5^{\prime}\right.$-CGTA-3 $\left.3^{\prime}\right)$, downstream template $\left(5^{\prime}\right.$-CGGCAT- $\left.3^{\prime}\right)$, and a $5^{\prime}$ phosphorylated downstream primer ( $5^{\prime}$-pGCCG-3'). Oligonucleotides comprising the upstream and downstream duplexes were separately mixed in equimolar ratios in $100 \mathrm{~mm}$ Tris, $\mathrm{pH} 7.5$, and $40 \mathrm{~mm} \mathrm{MgCl}_{2}$. Upstream and downstream DNA mixtures were separately annealed in a thermal cycler by denaturation at $94^{\circ} \mathrm{C}$, followed by a slow temperature gradient from $90^{\circ} \mathrm{C}$ to $4{ }^{\circ} \mathrm{C}$. The annealed DNA was then serially mixed in a 3:1 molar ratio with concentrated $\mathrm{hPol} \mu \Delta 2$ (7.5-11.3 $\left.\mathrm{mg} \mathrm{mL}^{-1}\right)$-first the downstream DNA, followed by the addition of the upstream DNA, and finally the incoming dUMPNPP nucleotide $(0.91 \mathrm{~mm}$ final concentration). The complex was incubated on ice at $4^{\circ} \mathrm{C}$ for $1 \mathrm{~h}$ after each addition. Crystals of the quaternary complex were grown at $4{ }^{\circ} \mathrm{C}$, by mixing equal volumes of complex and mother liquor (40-45.5 mM MES pH 5.6, 0.16-0.182 м KCl, 8.2-9.1 $\mathrm{mm} \mathrm{MgSO}_{4}, 8.2-9.1 \% \mathrm{w} / \mathrm{v}$ PEG400) using the sitting-drop vapor diffusion technique $^{33}$. Crystals were transferred to a cryoprotectant solution containing $40 \mathrm{~mm}$ MES pH 5.5, $50 \mathrm{~mm} \mathrm{NaCl}, 12 \mathrm{~mm} \mathrm{MgCl}_{2}, 0.1 \mathrm{~m} \mathrm{KCl}, 30 \%$ w/v PEG400, $8 \mathrm{~mm}$ $\mathrm{MgSO}_{4}, 10 \%$ glycerol, $1 \mathrm{~mm}$ dUMPNPP in two steps (PDB ID code 6WIC). The crystals were then flash frozen in liquid nitrogen and placed into a stream of nitrogen gas cooled to $-180^{\circ} \mathrm{C}$ for data collection.

Nucleotide exchange and incorporation in crystallo. For the post-catalytic complexes, quaternary complex crystals were transferred from cryoprotectant solution containing $1 \mathrm{~mm}$ dUMPNPP to cryoprotectant solution containing $10 \mathrm{~mm}$ dTTP and soaked for 22 hours (PDB ID code 6WID) or 47 hours (PDB ID code $6 \mathrm{WIE}$ ) at $4{ }^{\circ} \mathrm{C}$. The crystals were then flash frozen in liquid nitrogen and placed into a stream of nitrogen gas cooled to $-180^{\circ} \mathrm{C}$ for data collection.

Structure solution and refinement. Data were collected on the Southeast Regional Collaborative Access Team (SER-CAT) 22-ID beamline at the Advanced Photon Source (APS) at Argonne National Laboratory. The data were integrated and scaled using HKL $2000^{34}$. The crystal structure of the 1nt-gapped SSB ternary complex with an incoming nonhydrolyzable analog (incoming dUMPNPP opposite tem-

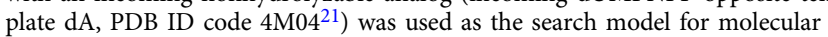
replacement in Phaser ${ }^{35}$. The same Rfree test reflections were used for all structures to avoid potential model bias. All structures were refined by iterative cycles of manual model building and refinement in COOT ${ }^{36,37}$ and Phenix ${ }^{38}$. TLS (Translation/Libration/Screw) vibrational motion refinement ${ }^{39}$ was used for all structures. Data collection and refinement statistics are listed in Table 1. Ramachandran statistics were generated using MolProbity ${ }^{40}$

NHEJ assays. pFASTBAC1 constructs of human Ku70H6 (C-terminal hexahistidine tag), Ku83, hLigIVH6, and XRCC $4^{41}$ were used to prepare baculovirus isolates for expression. All of the above constructs are available through Addgene. Hi5 cells were co-infected with isolates of either hLigIVH6 and XRCC4 (1:1 ratio) or Ku heterodimer (1:2 ratio of Ku70H6 to Ku83) constructs. Protein extracts were obtained in $50 \mathrm{~mm}$ sodium phosphate $\mathrm{pH} 8.0,1 \mathrm{M} \mathrm{KCl}, 10 \%$ glyercol, $0.25 \%$ Triton $\mathrm{X}-100,10 \mathrm{~mm}$ imidazole, and $7 \mathrm{~mm} \beta$-mercaptoethanol, loaded onto a Ni-NTA Superflow column, and step-eluted with extraction buffer containing $350 \mathrm{~mm}$ imidazole. The LigIV-XRCC4 and Ku complexes were dialyzed against $25 \mathrm{~mm}$ Tris $\mathrm{pH} 8,150 \mathrm{~mm} \mathrm{KCl}, 10 \%$ glycerol, and $2 \mathrm{~mm}$ DTT (Buffer A), bound to a Mono Q HR 5/5 column, and eluted using 20 column volumes (CV) linear gradient with 25 mм Tris pH 8, $400 \mathrm{~mm} \mathrm{KCl,} \mathrm{10 \%} \mathrm{glycerol,} 1 \mathrm{~mm}$ DTT (Buffer B). Purified Ku and LigIV-XRCC4 complexes were dialyzed against Buffer B for storage.

pRSET-B-hPol $\lambda^{42}$ was expressed in BL21-CodonPlus(DE3)-RIL cells, grown in $\mathrm{LB}$ medium at $28^{\circ} \mathrm{C}$ to an $\mathrm{OD}_{600 \mathrm{~nm}}$ of 0.5 . Protein expression was induced by addition of $1 \mathrm{mM}$ IPTG, followed 20 minutes later by $120 \mu \mathrm{gL}^{-1}$ rifampicin, and continued for 2 hours at $28^{\circ} \mathrm{C}$. Cells were pelleted by centrifugation, weighed, and frozen at $-20^{\circ} \mathrm{C}$. The frozen cell pellets were thawed and ground with 5.5 -fold wetweight of alumina in Buffer C ( $25 \mathrm{~mm}$ Tris pH 7.5, $1 \mathrm{M} \mathrm{NaCl}, 10 \%$ glycerol, $0.5 \mathrm{~mm}$ EDTA, $1 \mathrm{~mm}$ DTT) for 20 minutes at $4^{\circ} \mathrm{C}$. The suspension was clarified by centrifugation. DNA contamination was removed by precipitation with $0.3 \%$ polyethyleneimine and a second centrifugation step. The resulting supernatant was diluted four-fold to $250 \mathrm{~mm} \mathrm{NaCl}$ with Buffer D (25 mм Tris $\mathrm{pH} 7.5,10 \%$ glycerol, $0.5 \mathrm{~mm}$ EDTA, $1 \mathrm{~mm}$ DTT) and precipitated with ammonium sulfate to $65 \%$ saturation. The pellet was resuspended with Buffer D supplemented with $50 \mathrm{~mm}$ $\mathrm{NaCl}$ and loaded onto a PC column equilibrated with the same buffer. After copious washing with Buffer D supplemented with $100 \mathrm{~mm} \mathrm{NaCl}$, bound Pol $\lambda$ protein was eluted with Buffer D supplemented with $200 \mathrm{~mm} \mathrm{NaCl}$. The eluate was diluted with an equal volume of Buffer E (50 mM Tris $\mathrm{pH} 7.5,10 \%$ glycerol) and reloaded onto a PC column equilibrated with Buffer D supplemented with $100 \mathrm{~mm}$ $\mathrm{NaCl}$. After copious washing with Buffer E supplemented with $100 \mathrm{~mm} \mathrm{NaCl}$, the protein was eluted with Buffer F (20 mм phosphate buffer pH 7.8, $500 \mathrm{~mm} \mathrm{NaCl}$ ) and loaded onto a Ni-NTA column equilibrated with the same buffer. The bound protein was washed with Buffer F supplemented with increasing concentrations of imidazole, followed by elution with Buffer F containing $400 \mathrm{~mm}$ imidazole.

Fractions containing Pold were diluted five-fold with Buffer D, reloaded onto a PC column, and eluted with Buffer D supplemented with $500 \mathrm{~mm} \mathrm{NaCl}$. The resulting protein solution was adjusted by addition of glycerol and bovine serum albumin to $50 \%$, and $0.1 \mathrm{mg} \mathrm{mL}^{-1}$, respectively.

pRSET-Pol $\beta^{43}$ was expressed in BL21(DE3)-pLysS cells in LB medium at $37^{\circ} \mathrm{C}$ to an $\mathrm{OD}_{600 \mathrm{~nm}}$ of 0.5 . Protein expression was induced by addition of $1 \mathrm{~mm} \mathrm{IPTG}$ and continued at $37^{\circ} \mathrm{C}$ for 3 hours. Cells were pelleted by centrifugation and frozen at $-80^{\circ} \mathrm{C}$. The cell pellets were thawed and resuspended in Buffer G $(25 \mathrm{~mm}$ Tris- $\mathrm{HCl}$ pH 7.5, 1 mм EDTA, $1 \mathrm{~mm}$ PMSF, $10 \mathrm{~mm} \mathrm{Na}_{2} \mathrm{~S}_{2} \mathrm{O}_{5}$, and $1 \mathrm{mg} \mathrm{L}^{-1}$ pepstatin A) supplemented with $500 \mathrm{~mm} \mathrm{NaCl}$, lysed by sonication, and clarified by centrifugation. The resulting supernatant was diluted with Buffer $\mathrm{H}$ (Buffer G supplemented with 75 $\mathrm{mM} \mathrm{NaCl}$ ) and loaded onto a Q-sepharose column connected in series with a ssDNAcellulose column equilibrated with Buffer $\mathrm{H}$. Pol $\beta$ passes through the Q-sepharose and is retained in the ssDNA-cellulose matrix. After loading, the Q-sepharose column was detached from the ssDNA-cellose, at which point the ssDNA-cellulose is further washed with Buffer $\mathrm{H}$, and bound proteins are eluted by a linear gradient to Buffer I (Buffer $\mathrm{G}$ supplemented with $1 \mathrm{M} \mathrm{NaCl}$ ). Fractions containing Pol $\beta$ were dialyzed against Buffer $\mathrm{H}$, passed through a $0.22 \mu \mathrm{m}$ filter and loaded onto a Mono S HR 10/10 column equilibrated with Buffer $\mathrm{H}$. Bound proteins were eluted with a linear gradient to $1 \mathrm{M} \mathrm{NaCl}$ (Buffer I).

Both 300 bp DNA substrates were generated by PCR amplification of a fragment of the mouse Jk1 locus in the presence of Cy5-labeled dCTP, followed by restriction digestion of sites appended to this common core. The $3^{\prime}$ GCG overhang-containing substrate (complementary DSB) was generated by amplification with primers $5^{\prime}$-TTT TTGCCACGCTGGCTTAGCTGTATAGTCAGGGA-3' and 5'-CACCTGCCTCGC TGGCACACCCATCTCAGACTGGC-3', and digested with BglI. The 3'-G overhang substrate (noncomplementary DSB) was generated by amplification with primers 5'-CAAGTGGACCACATGTCTTAGCTGTATAGTCAGGGAAATC-3' and 5'-CCG CCGACGCCATGTCACACCCATCTCAGACTGGCTACCC-3', and digested with AhdI. Complete digestion was validated by electrophoresis, and substrates further purified with a QIAquick cartridge. End-joining reactions were performed with $1 \mathrm{~nm}$ substrate, $10 \mathrm{~nm} \mathrm{Ku}, 20 \mathrm{~nm}$ XRCC4-Ligase IV complex, and $12.5 \mathrm{~nm}$ polymerase in a reaction with $10 \%$ polyethylene glycol, $150 \mathrm{~mm} \mathrm{KCl}, 25 \mathrm{~mm}$ Tris $\mathrm{pH}$ 7.5, $10 \mu \mathrm{m}$ each dNTP, $100 \mu \mathrm{m}$ each rNTP, and $100 \mathrm{ng}$ plasmid DNA for 10 minutes. Ligation products were resolved using 5\% native PAGE electrophoresis before gel imaging and qualitative analysis.

Reporting summary. Further information on research design is available in the Nature Research Reporting Summary linked to this article.

\section{Data availability}

Atomic coordinates and structure factors have been deposited in the Protein Data Bank (www.pdb.org) with ID codes 6WIC [https://doi.org/10.2210/pdb6WIC/pdb], 6WID [https://doi.org/10.2210/pdb6WID/pdb], and 6WIE [https://doi.org/10.2210/pdb6WIE/ $\mathrm{pdb}]$. Source data are provided with this paper. Other data supporting the findings of this study are available from the corresponding author upon reasonable request. Source data are provided with this paper.

Received: 16 June 2020; Accepted: 17 August 2020;

Published online: 22 September 2020

\section{References}

1. von Sonntag, C. Free-radical-induced DNA damage and its repair, (SpringerVerlag, Heidelberg, 2006)

2. Jackson, S. P. \& Bartek, J. The DNA-damage response in human biology and disease. Nature 461, 1071-1078 (2009).

3. Schatz, D. G. \& Swanson, P. C. V(D)J recombination: mechanisms of initiation. Annu. Rev. Genet. 45, 167-202 (2011).

4. Takata et al. Homologous recombination and non-homologous end-joining pathways of DNA double-strand break repair have overlapping roles in the 
maintenance of chromosomal integrity in vertebrate cells. EMBO J. 17, 5497-5508 (1998).

5. Lieber, M. R. The mechanism of double-strand DNA break repair by the nonhomologous DNA end-joining pathway. Annu. Rev. Biochem. 79, 181-211 (2010).

6. Moon et al. The X family portrait: structural insights into biological functions of X family polymerases. DNA Repair (Amst.) 6, 1709-1725 (2007).

7. Bertocci, B. et al. Immunoglobulin kappa light chain gene rearrangement is impaired in mice deficient for DNA polymerase mu. Immunity 19, 203-211 (2003).

8. Bertocci, B., De Smet, A., Weill, J. C. \& Reynaud, C. A. Nonoverlapping functions of DNA polymerases mu, lambda, and terminal deoxynucleotidyltransferase during immunoglobulin $\mathrm{V}(\mathrm{D}) \mathrm{J}$ recombination in vivo. Immunity 25, 31-41 (2006).

9. Lieber, M. R. The polymerases for V(D)J recombination. Immunity 25, 7-9 (2006).

10. Gilfillan, S., Benoist, C. \& Mathis, D. Mice lacking terminal deoxynucleotidyl transferase: adult mice with a fetal antigen receptor repertoire. Immunol. Rev. 148, 201-219 (1995).

11. Mahajan, K. N., Nick McElhinny, S. A., Mitchell, B. S. \& Ramsden, D. A. Association of DNA polymerase mu (pol $\mathrm{mu}$ ) with $\mathrm{Ku}$ and ligase IV: role for pol mu in end-joining double-strand break repair. Mol. Cell Biol. 22, 5194-5202 (2002).

12. Nick McElhinny, S. A. et al. A gradient of template dependence defines distinct biological roles for family X polymerases in nonhomologous end joining. Mol. Cell 19, 357-366 (2005).

13. Braithwaite et al. DNA polymerase lambda mediates a back-up base excision repair activity in extracts of mouse embryonic fibroblasts. J. Biol. Chem. 280, 18469-18475 (2005).

14. Brown, J. A., Pack, L. R., Sanman, L. E. \& Suo, Z. Efficiency and fidelity of human DNA polymerases lambda and beta during gap-filling DNA synthesis. DNA Repair (Amst.) 10, 24-33 (2011).

15. Nick McElhinny, S. A. \& Ramsden, D. A. Polymerase mu is a DNA-directed DNA/RNA polymerase. Mol. Cell Biol. 23, 2309-2315 (2003).

16. Roettger, M. P., Fiala, K. A., Sompalli, S., Dong, Y. \& Suo, Z. Pre-steady-state kinetic studies of the fidelity of human DNA polymerase mu. Biochemistry 43, 13827-13838 (2004).

17. Garcia-Diaz, M., Bebenek, K., Krahn, J. M., Kunkel, T. A. \& Pedersen, L. C. A closed conformation for the Pol lambda catalytic cycle. Nat. Struct. Mol. Biol. 12, 97-98 (2005)

18. Garcia-Diaz, M., Bebenek, K., Krahn, J. M., Pedersen, L. C. \& Kunkel, T. A. Role of the catalytic metal during polymerization by DNA polymerase lambda. DNA Repair (Amst.) 6, 1333-1340 (2007).

19. Moon et al. Structural insight into the substrate specificity of DNA Polymerase mu. Nat. Struct. Mol. Biol. 14, 45-53 (2007).

20. Moon, A. F., Gosavi, R. A., Kunkel, T. A., Pedersen, L. C. \& Bebenek, K. Creative template-dependent synthesis by human polymerase mu. Proc. Natl Acad. Sci. USA 112, E4530-E4536 (2015).

21. Moon et al. Sustained active site rigidity during synthesis by human DNA polymerase mu. Nat. Struct. Mol. Biol. 21, 253-260 (2014).

22. Gouge et al. Structural basis for a novel mechanism of DNA bridging and alignment in eukaryotic DSB DNA repair. EMBO J. 34, 1126-1142 (2015).

23. Loc'h, J., Rosario, S. \& Delarue, M. Structural basis for a new templated activity by terminal deoxynucleotidyl transferase: implications for $\mathrm{V}(\mathrm{D}) \mathrm{J}$ recombination. Structure 24, 1452-1463 (2016).

24. Loc'h et al. Structural evidence for an in trans base selection mechanism involving Loop1 in polymerase mu at an NHEJ double-strand break junction. J. Biol. Chem. 294, 10579-10595 (2019).

25. Zhao, B., Watanabe, G. \& Lieber, M. R. Polymerase mu in non-homologous DNA end joining: importance of the order of arrival at a double-strand break in a purified system. Nucleic Acids Res. 48, 3605-3618 (2020).

26. Sastre-Moreno et al. Polmu tumor variants decrease the efficiency and accuracy of NHEJ. Nucleic Acids Res. 45, 10018-10031 (2017).

27. Cancer Genome Atlas Research, N. Integrated genomic analyses of ovarian carcinoma. Nature 474, 609-615 (2011).

28. Durinck et al. Temporal dissection of tumorigenesis in primary cancers. Cancer Discov. 1, 137-143 (2011).

29. Martin, M. J., Juarez, R. \& Blanco, L. DNA-binding determinants promoting NHEJ by human Polmu. Nucleic Acids Res. 40, 11389-11403 (2012).

30. Jamsen et al. Time-lapse crystallography snapshots of a double-strand break repair polymerase in action. Nat. Commun. 8, 253 (2017).

31. Davis, B. J., Havener, J. M. \& Ramsden, D. A. End-bridging is required for pol $\mathrm{mu}$ to efficiently promote repair of noncomplementary ends by nonhomologous end joining. Nucleic Acids Res. 36, 3085-3094 (2008).

32. Martin, M. J. \& Blanco, L. Decision-making during NHEJ: a network of interactions in human Polmu implicated in substrate recognition and endbridging. Nucleic Acids Res. 42, 7923-7934 (2014).
33. Chayen, N. E. Comparative studies of protein crystallization by vapourdiffusion and microbatch techniques. Acta Crystallogr. D. Biol. Crystallogr. 54, 8-15 (1998)

34. Otwinowski, Z. M. W. Processing of X-ray diffraction data collected in oscillation mode. Methods Enzymol. 276, 307-326 (1997).

35. Zwart et al. Automated structure solution with the PHENIX suite. Methods Mol. Biol. 426, 419-435 (2008).

36. Emsley, P. \& Cowtan, K. Coot: model-building tools for molecular graphics. Acta Crystallogr. D. Biol. Crystallogr. 60, 2126-2132 (2004).

37. Emsley, P., Lohkamp, B., Scott, W. G. \& Cowtan, K. Features and development of Coot. Acta Crystallogr. D. Biol. Crystallogr. 66, 486-501 (2010).

38. Adams et al. PHENIX: a comprehensive python-based system for macromolecular structure solution. Acta Crystallogr. D. Biol. Crystallogr. 66, 213-221 (2010).

39. Urzhumtsev, A., Afonine, P. V. \& Adams, P. D. TLS from fundamentals to practice. Crystallogr. Rev. 19, 230-270 (2013).

40. Lovell et al. Structure validation by calpha geometry: phi,psi and Cbeta deviation. Proteins 50, 437-450 (2003).

41. Nick McElhinny, S. A., Snowden, C. M., McCarville, J. \& Ramsden, D. A. Ku recruits the XRCC4-ligase IV complex to DNA ends. Mol. Cell Biol. 20, 2996-3003 (2000).

42. Garcia-Diaz et al. DNA polymerase lambda, a novel DNA repair enzyme in human cells. J. Biol. Chem. 277, 13184-13191 (2002).

43. Prasad, R., Kumar, A., Widen, S. G., Casas-Finet, J. R. \& Wilson, S. H. Identification of residues in the single-stranded DNA-binding site of the 8$\mathrm{kDa}$ domain of rat DNA polymerase beta by UV cross-linking. J. Biol. Chem. 268, 22746-22755 (1993).

\section{Acknowledgements}

We thank J. Krahn for nucleotide parameter optimization, and W. Beard and R. Stanley for critical reading of the manuscript. This work was supported in part by Division of Intramural Research of the National Institute of Environmental Health Sciences, National Institutes of Health Grants 1ZIA ES102645 (to L.C.P.) and Z01 ES065070 (to T. A.K.), and National Cancer Institute grant CA097096 (to D.A.R). Use of the Advanced Photon Source was supported by the US Department of Energy, Office of Science, Office of Basic Energy Sciences [W-31-109-Eng-38].

\section{Author contributions}

A.M.K. and J.M.P. designed and performed research; K.B. contributed materials; A.M.K., J.M.P., D.A.R., T.A.K., L.C.P., and K.B. contributed to data analysis and manuscript preparation.

\section{Competing interests}

The authors declare no competing interests.

\section{Additional information}

Supplementary information is available for this paper at https://doi.org/10.1038/s41467020-18506-5.

Correspondence and requests for materials should be addressed to L.C.P.

Peer review information Nature Communications thanks the anonymous reviewer(s) for their contribution to the peer review of this work.

Reprints and permission information is available at http://www.nature.com/reprints

Publisher's note Springer Nature remains neutral with regard to jurisdictional claims in published maps and institutional affiliations.

Open Access This article is licensed under a Creative Commons Attribution 4.0 International License, which permits use, sharing, adaptation, distribution and reproduction in any medium or format, as long as you give appropriate credit to the original author(s) and the source, provide a link to the Creative Commons license, and indicate if changes were made. The images or other third party material in this article are included in the article's Creative Commons license, unless indicated otherwise in a credit line to the material. If material is not included in the article's Creative Commons license and your intended use is not permitted by statutory regulation or exceeds the permitted use, you will need to obtain permission directly from the copyright holder. To view a copy of this license, visit http://creativecommons.org/ licenses/by/4.0/.

This is a U.S. government work and not under copyright protection in the U.S.; foreign copyright protection may apply 2020 